OPEN ACCESS

Edited by:

Prasanna K. Santhekadur. JSS Academy of Higher Education and Research, India

Reviewed by: Wafaa M. Rashed, Children's Cancer Hospital, Egypt Michael Bordonaro, Geisinger Commonwealth School of Medicine, United States

${ }^{*}$ Correspondence: Hong-Mei Yang yanghm_z@@126.com

Specialty section: This article was submitted to Gastrointestinal Cancers, a section of the journal

Frontiers in Oncology

Received: 30 November 2020 Accepted: 11 May 2021 Published: 03 June 2021

Citation:

Liang Y, Ma B, Jiang P and Yang H-M (2021) Identification of MethylationRegulated Differentially Expressed Genes and Related Pathways in Hepatocellular Carcinoma: A Study Based on TCGA Database and Bioinformatics Analysis.

Front. Oncol. 11:636093. doi: 10.3389/fonc.2021.636093

\section{Identification of Methylation- Regulated Differentially Expressed Genes and Related Pathways in Hepatocellular Carcinoma: A Study Based on TCGA Database and Bioinformatics Analysis}

\author{
Yu Liang ${ }^{1}$, Bin Ma ${ }^{1}$, Peng Jiang ${ }^{1}$ and Hong-Mei Yang ${ }^{2 *}$ \\ 1 Department of Colorectal Surgery, Cancer Hospital of China Medical University, Liaoning Cancer Hospital \& Institute, \\ Shenyang, China, ${ }^{2}$ Department of Internal Oncology, Cancer Hospital of China Medical University, Liaoning Cancer Hospital \\ \& Institute, Shenyang, China
}

Background: In recent years, DNA methylation modification has been shown to be a critical mechanism in the field of epigenetics.

Methods: Hepatocellular carcinoma (HCC) data were obtained from The Cancer Genome Atlas project, including RNA expression profiles, Illumina Human Methylation 450K BeadChip data, clinical information, and pathological features. Then, differentially expressed genes (DEGs) and differentially methylated genes were identified using $R$ software. Methylation-regulated DEGs (MeDEGs) were further analyzed using Spearman's correlation analysis. Gene ontology (GO) and Kyoto Encyclopedia of Genes and Genomes (KEGG) pathway analyses were performed using the DAVID database and ClueGO in Cytoscape software. Kaplan-Meier survival analysis explored the relationship between methylation, expression of MeDEGs, and survival time. Gene set enrichment analysis (GSEA) was conducted to predict the function of prognosis-related MeDEGs.

Results: A total of nine up-regulated and 72 down-regulated MeDEGs were identified. GO and KEGG pathway analyses results indicated that multiple cancer-related terms were enriched. Kaplan-Meier survival analysis showed that the methylation status of four MeDEGs (CTF1, FZD8, PDK4, and ZNF334) was negatively associated with overall survival. Moreover, the methylation status of CDF1 and PDK4 was identified as an independent prognostic factor. According to GSEA, hypermethylation of prognosisrelated MeDEGs was enriched in pathways that included "Spliceosome", "Cell cycle", "RNA degradation", "RNA polymerase", "DNA replication", "Mismatch repair", "Base excision repair", "Nucleotide excision repair", "Homologous recombination", "Protein export", and "Pyrimidine metabolism". 
Conclusions: Aberrant DNA methylation plays a critical role in malignant progression of HCC. Prognosis-related MeDEGs identified in this research may be potential biomarkers and targets in diagnosis and treatment.

Keywords: hepatocellular carcinoma, DNA methylation, overall survival, bioinformatic analysis, methylation-regulated differentially expressed genes

\section{INTRODUCTION}

Hepatocellular carcinoma (HCC) is the most common histopathological type of liver cancer, which ranked seventh in incidence and third in mortality among tumors worldwide in 2018 (1). Epidemiological studies have confirmed that HCC occurrence is associated with chronic hepatitis $\mathrm{B} / \mathrm{C}$ virus infection, liver cirrhosis, environmental toxins, non-alcoholic fatty liver disease, metabolic disease, and lifestyle factors $(2,3)$. Although surgery combined with chemotherapy, radiotherapy, and immunotherapy can improve patient prognosis, the fiveyear survival rate in advanced-stage patients is still $<15 \%$ (4). Hence, a study into crucial biomarkers and molecular therapeutic pathways is of great significance for improving HCC patient prognosis.

DNA methylation modification has been considered to be a critical gene regulation mechanism in epigenetics and has been verified to be a reversible process. In the genome of normal cells, promoter cytosine-phosphate-guanine $(\mathrm{CpG})$-islands are typically hypomethylated. However, tumor cell hypermethylation of the CpG-island in the tumor suppressor promoter region is associated with malignant formation and progression. For instance, zinc finger protein 382 (ZNF382) is a potent tumor-suppressor and is down-regulated in hepatitis B-related HCC due to promoter methylation (5). However, research into DNA methylation of individual genes and pathways remains insufficient. Screening methylation-regulated differentially expressed genes (MeDEGs) with high-throughput data is of profound significance for clarifying the role of methylation and identifying future research directions.

In recent years, diverse gene-sequencing platforms have been utilized in basic and clinical HCC research. In addition, these techniques provide evidence for accurate tumor therapy. For instance, Illumina Human Methylation 450K BeadChip has been employed to detect genome-wide aberrant DNA methylation

\footnotetext{
Abbreviations: HCC, Hepatocellular carcinoma; CpG, Cytosine-phosphateguanine; MeDEGs, Methylation-regulated differentially expressed genes; TCGA, The Cancer Genome Atlas; GSEA, Gene set enrichment analysis; DEGs, Differentially expressed genes; DMGs, Differentially methylated genes; FC, Fold change; GO, Gene ontology; KEGG, Kyoto Encyclopedia of Genes and Genomes; Hyper-LG, hypermethylation and low-expression MeDEG; Hypo-HG, hypomethylation and high-expression MeDEG; ZNF334, Zinc finger protein 334; CTF1, Cardiotrophin-1; FZD8, Frizzled-8; PDK4, Pyruvate dehydrogenase kinase 4; KLF4, Kruppel-like factor 4; TACSTD2, Tumor-associated calcium signal transducer 2; RAC2, Ras-related C3 botulinum toxin substrate 2; GNB4, Guanine nucleotide-binding protein subunit beta-4; PTGER4, Prostaglandin E2 receptor EP4 subtype; CCNE2, G1/S-specific cyclin-E2; TGFB3, transforming growth factor beta-3; PRSAKA6, Ribosomal protein S6 kinase alpha-6; LAMA2, Laminin subunit alpha-2; GSTM1, Glutathione S-transferase Mu 1; MSI, Microsatellite-instability.
}

profiles between HCC cell line Huh7 and normal cell line L02. As a result, 62,702 (61.3\%) CpG-island sites were hypermethylated and 39,552 (38.7\%) CpG-island sites were hypomethylated (6). Zhang et al. have indicated that distinct DNA methylation differences emerge in the host immune system at an early stage based on the Illumina Human Methylation 450K BeadChip data, which may serve as noninvasive diagnostic HCC markers (7). The Illumina Methylation 450K BeadChip has been shown to play a critical role in the field of tumor epigenetics, but there is still a lack of conjoint correlation analysis of methylation, gene expression, and patient prognosis in large cohorts.

The present study applied bioinformatics analysis to identify MeDEGs based on in silico and clinical data from The Cancer Genome Atlas (TCGA, http://cancergenome.nih.gov) project (8). Then, MeDEG enrichment analysis was performed using an online database. Methylation of four genes was associated with prognosis in HCC patients. Gene set enrichment analysis (GSEA) was also performed.

\section{MATERIALS AND METHODS}

\section{Data Collection and MeDEG Identification}

TCGA database included the expression profiles of 374 HCC and 50 normal tissues (level 3) derived using RNA-seq and methylation data from $380 \mathrm{HCC}$ and 50 normal tissues analyzed with the Illumina Human Methylation 450K BeadChip platform up to March 2020. Genomic Data Commons Data Transfer Tool 1.3.0 (8) was used to download the above profiles and clinical information data for further analysis. This research conformed to the guidelines published by TCGA on December 2015 (https:// cancergenome.nih.gov/publications/publicationguidelines) and approval from an ethics committee was not required.

An RNA matrix that included 50 normal hepatic tissues and corresponding HCC tissues was constructed using PERL software. Methylation data matrix including 50 paired HCC and normal tissue samples was constructed using the same method. Differentially expressed genes (DEGs) and differentially methylated genes (DMGs) were identified using the "edgeR" and "limma" packages in R software with a threshold log2 fold change (FC) $>1.0$ and $\mathrm{P}<0.01$. After a total of 42 normal and 374 HCC tissues were analyzed using RNA-seq and Illumina Human Methylation $450 \mathrm{~K}$ BeadChip platform, expression and methylation data were merged together for Spearman's correlation analysis. The hypermethylated down-regulated and hypomethylated up-regulated genes that satisfied the cut-off criteria, including correlation coefficient $<0.2$ and $\mathrm{P}<0.01$, were identified as MeDEGs. Furthermore, a heat map of the top 100 
differentially expressed and methylated genes in 50 paired tissues were mapped using the "heatmap" package in R software.

\section{MeDEG Enrichment Analyses}

To further clarify the function of MeDEGs in HCC carcinogenesis and progression, gene ontology (GO) (9) and Kyoto Encyclopedia of Genes and Genomes (KEGG) pathways (10) analyses were performed using the DAVID database (https://david.ncifcrf.gov/) and ClueGO $(11,12)$ in Cytoscape 3.7.1. The enrichment results of GO and KEGG analyses were visualized as a bubble chart and network diagram, respectively. Differences with $\mathrm{P}<0.05$ were regarded as statistically significant.

\section{Association Analysis of MeDEGs and Patient Prognosis}

A total of 353 enrolled HCC patients were followed up for 80 months and had complete clinical data for the survival analysis. The 353 HCC patients were sorted into two groups according to the MeDEG median methylation value. In addition, a hypermethylation and low-expression MeDEG (Hyper-LG) group and a hypomethylation and high-expression MeDEG (Hypo-HG) group were established according to the median value of MeDEG methylation and expression. Kaplan-Meier method and log-rank test were used to compare the overall survival between the two groups using the "survival" package in $\mathrm{R}$ software. Differences with $\mathrm{P}<0.05$ were regarded as statistically significant.

\section{GSEA of Prognosis-Related MeDEGs}

GSEA of prognosis-related MeDEGs was conducted using GSEA 3.0 software with gene set c2 (cp.kegg.v.6.2.symbols.gmt). RNA expression profiles for $374 \mathrm{HCC}$ tissues were selected as the dataset. The sample was marked as either "Hypermethylation" or "Hypomethylation" based on the median methylation value of prognosis-related MeDEGs. The enrichment score $>0.4$ and $\mathrm{P}<$ 0.05 were regarded as statistically significant.

\section{Statistical Analysis}

All statistical analyses were performed using SPSS V18.0 (SPSS, Inc., Chicago, IL, USA). The association between methylation of prognosis-related MeDEGs and clinicopathological characteristics was analyzed using the chi-squared test. Cox proportional hazards model was applied to evaluate the influence of clinical data and methylation on prognosis. Differences with $\mathrm{P}<0.05$ were regarded as statistically significant.

\section{RESULTS}

\section{Identification of MeDEGs in HCC}

A total of 3157 up-regulated and 1080 down-regulated genes were screened as DEGs from 50 paired HCC and normal tissue samples. The top 100 DEGs with the highest and most significant differences are represented on a heat map in Figure 1A. Moreover, 1061 hypermethylated and 1401 hypomethylated DMGs were identified
A

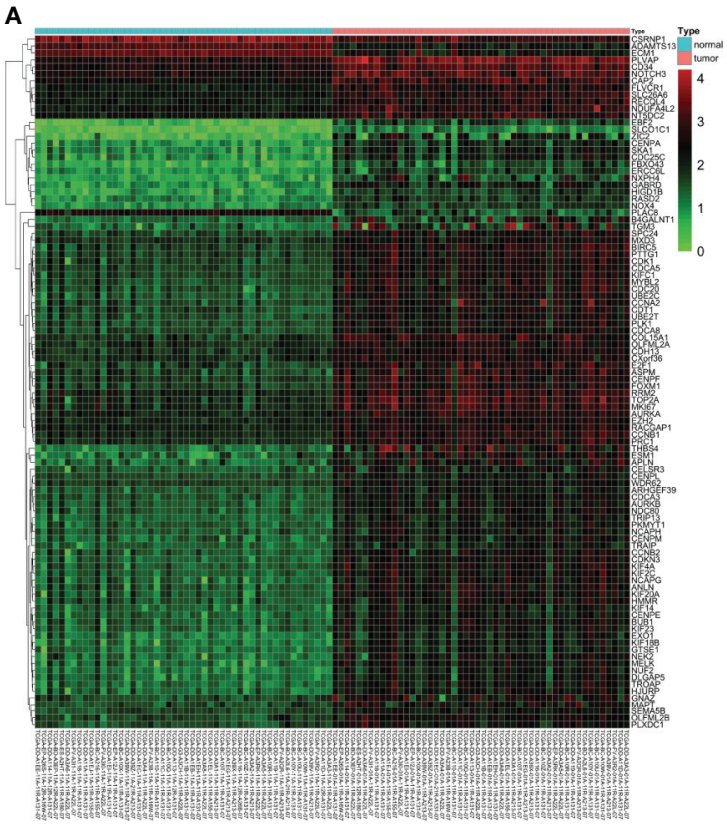

B

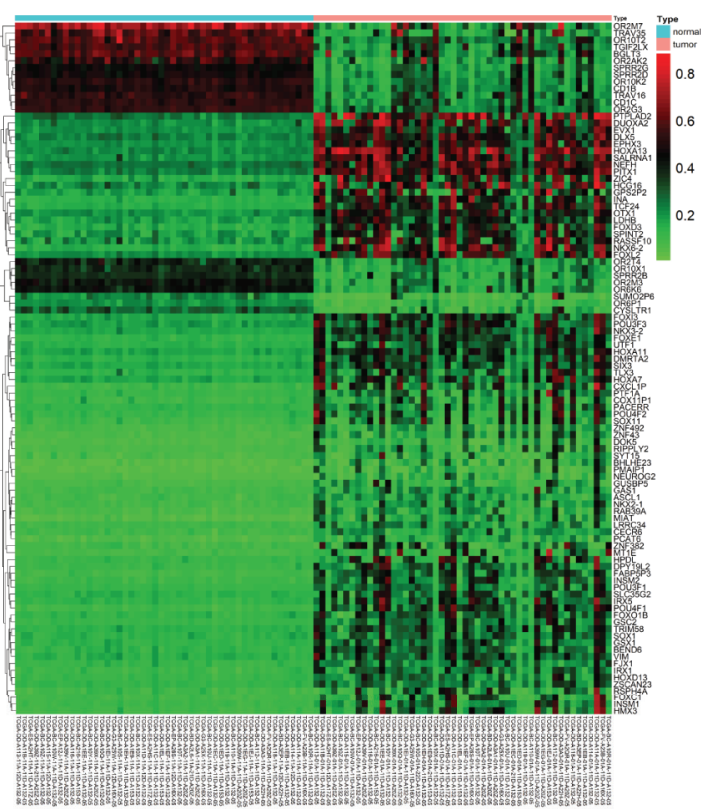

FIGURE 1 | Differentially expressed genes (DEGs) and differentially methylated genes (DMGs) identified from The Cancer Genome Atlas (TCGA) database. (A) Heat map of the top $100 \mathrm{DEGs}$ ( $\log _{2} \mathrm{FC}>2, P<0.01$ ). Lower horizontal axis marks sample names, left vertical axis shows clusters of DEGs, and right vertical axis represents gene names. Red represents up-regulated genes and green represents down-regulated genes. (B) Heat map of the top 100 DMGs (log 2 FC $>1$, $P<0.01)$. Lower horizontal axis marks sample names, left vertical axis shows clusters of DMGs, and right vertical axis represents gene names. Red represents hypermethylated genes and green represents hypomethylated genes. 
and represented as a heat map of the top 100 DMGs (Figure 1B). According to the Spearman's correlation analysis results, 359 genes had a negative correlation between expression and methylation. Nine up-regulated and 72 down-regulated MeDEGs that satisfied the three conditions were obtained and gene lists were also identified (Figure 2). The top ten MeDEGs with the highest Spearman's correlation coefficient are shown in Figure 3.

\section{Functional Enrichment Analyses of MeDEGs}

GO analysis was used to clarify the function of 81 MeDEGs using DAVID 6.8 software (Figure 4). The biological process and molecular function terms were mainly associated with transcription regulation. Moreover, negative regulation of cell proliferation and motility was also enriched. In addition, KEGG pathway analysis results indicated that "Pathways in cancer", "Inflammatory bowel disease", "Transcriptional misregulation in cancer", and "Malaria" were significantly involved in MeDEGs. "Hepatocellular carcinoma" was also enriched and KEGG network enrichment diagram was mapped in Figure 5.

\section{Prognosis-Related MeDEGs in HCC}

Kaplan-Meier curve analysis revealed a relationship between MeDEG methylation value and overall survival in HCC patients. Hypermethylation of cardiotrophin-1 (CTF1), Frizzled-8 (FZD8), pyruvate dehydrogenase kinase 4 (PDK4), and zinc finger protein 334 (ZNF334) was negatively correlated with the overall survival (Figures 6A-D). Then, prognosis of the above four MeDEGs was further compared with the Hyper-LG and Hypo-HG groups. As compared with patients in the Hyper-LG group, Hypo-HG patients had a significant better survival (Figures 6E-H).

\section{Identification of Methylation-Based Biomarkers}

A total of 353 patients were divided into "Low" and "High" groups according to the median methylation of CTF1, FZD8, PDK4, and ZNF334. CTF1 methylation status significantly correlated with gender and $\mathrm{T}$ stage. In addition, PDK4 hypermethylation was associated with gender, $\mathrm{T}$ stage, and pathologic stage (Table 1). Univariate and multivariate Cox regression analyses were then conducted to evaluate the prognostic role of the above four genes' methylation status. The samples were divided into high or low methylation groups according to the median gene methylation status. Advanced $\mathrm{T}$ stage, pathologic stage, and high methylation of prognosisrelated MeDEGs were associated with poor HCC patient prognosis (Table 2). Multivariate analysis results identified $\mathrm{T}$ stage and methylation status of CTF1 and PDK4 as independent factors in the overall survival.

\section{GSEA of Prognosis-Related MeDEGs}

GSEA results revealed the potential mechanisms of prognosisrelated MeDEGs. A total of 11 consensus terms were obtained from the enriched KEGG terms and included "Spliceosome", "Cell cycle", "RNA degradation", "RNA polymerase", "DNA replication", "Mismatch repair", "Base excision repair", "Nucleotide excision repair", "Homologous recombination", "Protein export", and "Pyrimidine metabolism" (Figure 7A). PDK4 enrichment is represented as an example in Figure $\mathbf{7 B}$.

\section{DISCUSSION}

Accumulating evidence has indicated that aberrant DNA methylation modification is a critical molecular event in HCC progression. Hypermethylated status in the promoter of tumor suppressor genes (13), cyclin (14), and DNA mismatch genes (15) has been studied in depth. HCC cell methylation profiles have become a new field of tumor biomarker study (16). Furthermore, DNA methylation has been recognized as a potential therapeutic target due to its reversibility (17). Therefore, MeDEG identification will provide more information on the role of methylation in HCC.

Bioinformatics analysis in the present study resulted in 81 MeDEGs. GO analysis indicated that the main terms are related
A

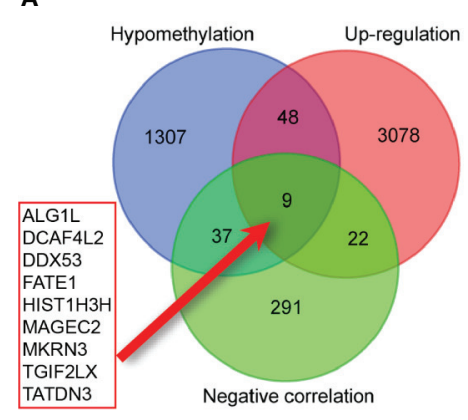

B

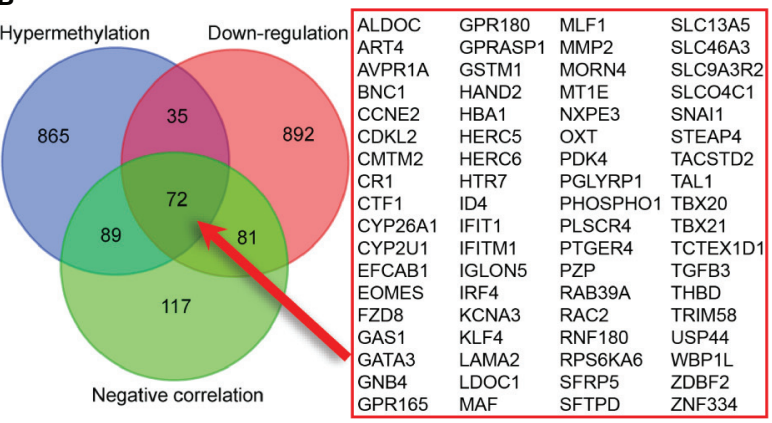

FIGURE 2 | Identification of methylation-regulated differentially expressed genes (MeDEGs). (A) A total of nine genes were identified as MeDEGs by intersecting three gene sets (hypomethylation, up-regulation, and negative correlation). (B) A total of 72 genes were identified as MeDEGs by intersecting three gene sets (hypermethylation, down-regulation, and negative correlation). 

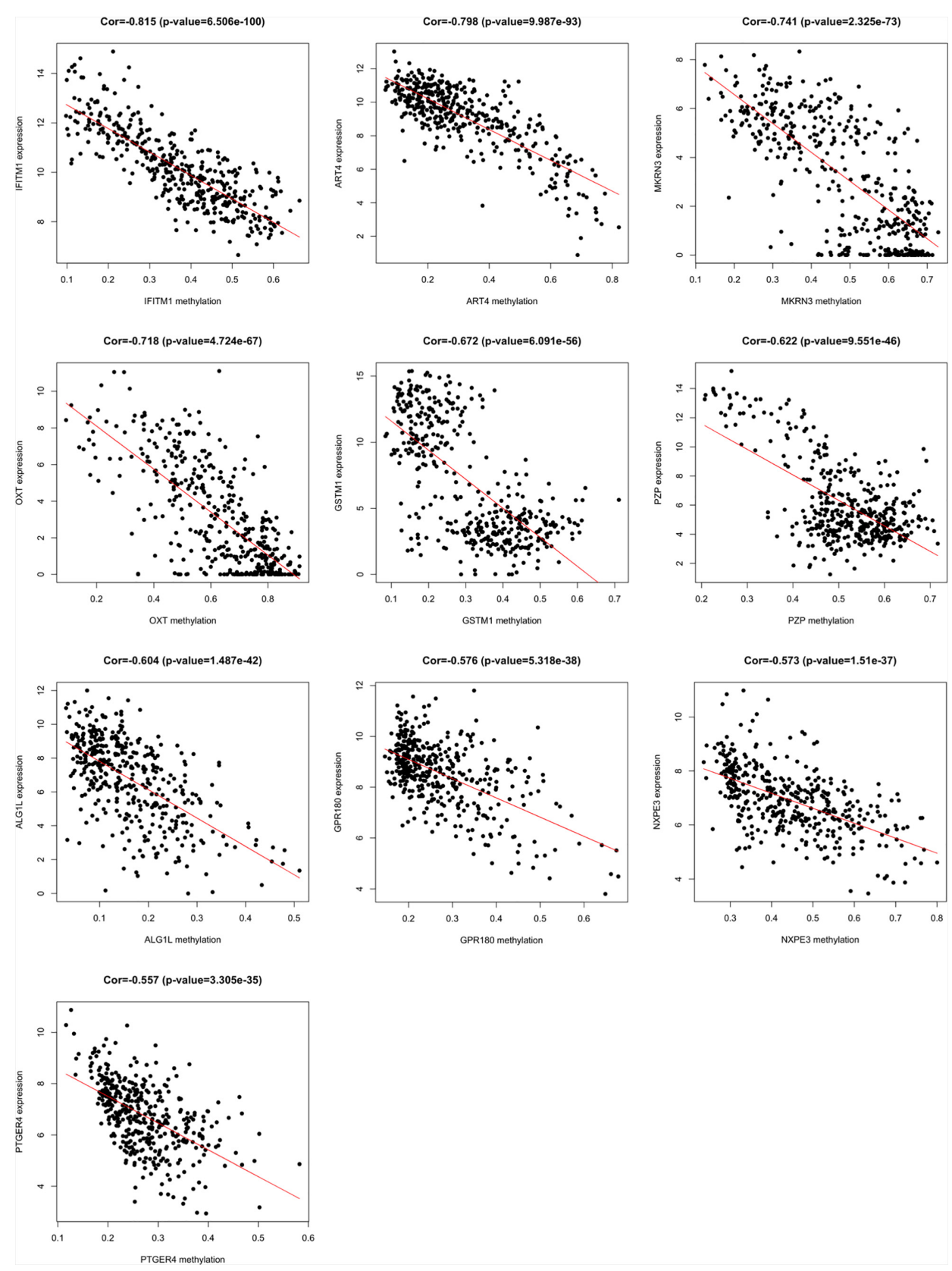

FIGURE 3 | Methylation-regulated differentially expressed genes (MeDEGs) with the top ten correlation coefficients. Spearman's correlation analysis for methylation (horizontal axis) and expression (vertical axis) of MeDEGs. Spearman's correlation coefficient and P-values are shown in each plot.

to transcription dysregulation. For instance, Kruppel-like factor 4 (KLF4) has been identified as a transcription factor that can suppress the expression of Ring1- and YY1-binding protein and inhibit HCC tumorigenesis (18). Moreover, KLF4 expression was epigenetically inhibited by CpG-island hypermethylation (19). Therefore, it was speculated that methylation can indirectly control gene expression by regulating transcription factors. The present study also identified tumor-associated calcium signal transducer 2 (TACSTD2) as a MeDEG, which has been reported to be down-regulated in primary HCC tissue. However, no research has demonstrated that TACSTD2 is regulated by methylation in HCC, which will be the subject of future studies. KEGG pathway analysis further defined the role of MeDEGs in HCC. It is noteworthy that "Pathways in cancer" was the most enriched pathway in which Ras-related C3 botulinum toxin substrate 2 (RAC2), guanine nucleotidebinding protein subunit beta-4 (GNB4), prostaglandin E2 receptor EP4 subtype (PTGER4), G1/S-specific cyclin-E2 


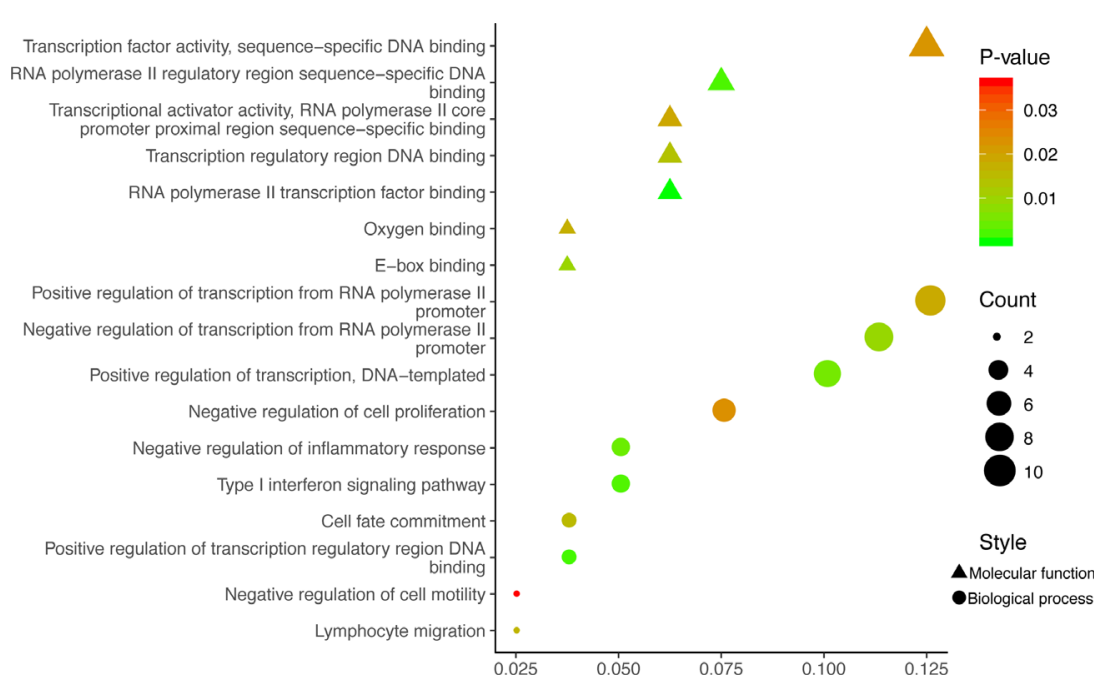

FIGURE 4 | MeDEG gene ontology enrichment analysis. Molecular function and biological process terms for MeDEGs are shown as "triangles" and "circles", respectively. "Count" represents the number of genes. MeDEGs, methylation-regulated differentially expressed genes.

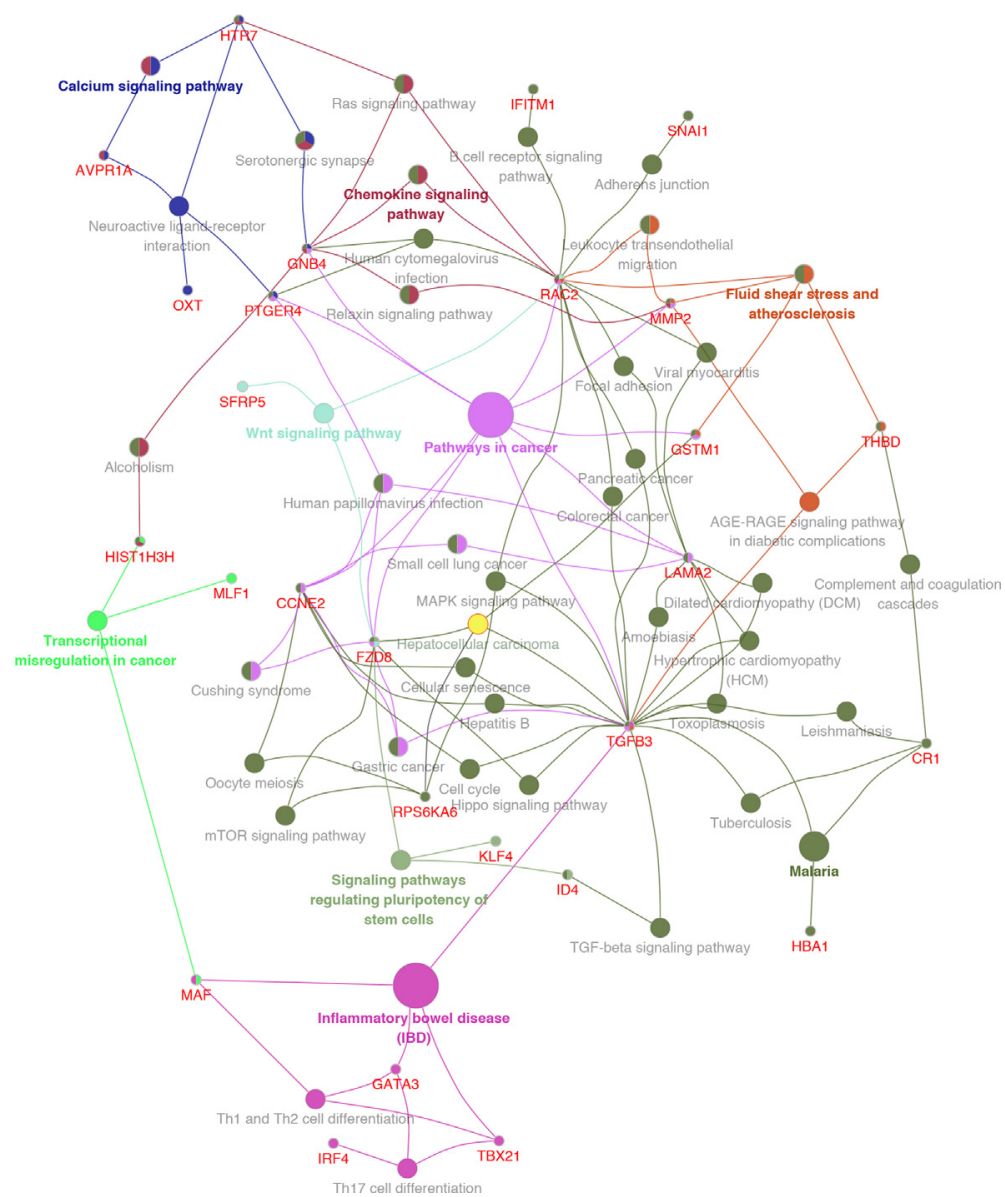

FIGURE 5 | KEGG pathway enrichment network diagram for MeDEGs. MeDEGs and enrichment pathways constitute a regulatory network. The larger the pathway circle, the more genes were enriched. 


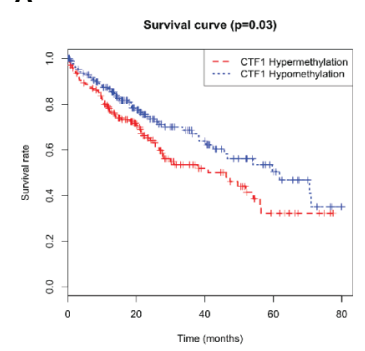

E

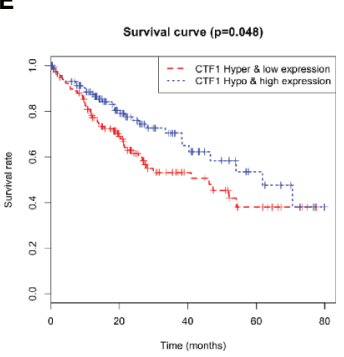

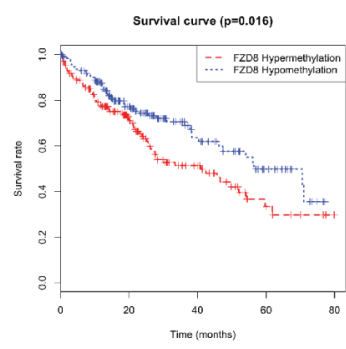

$\mathbf{F}$

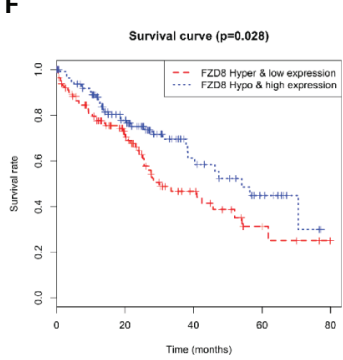

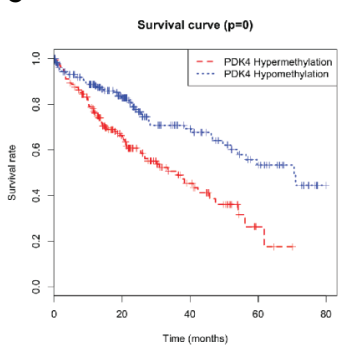

G

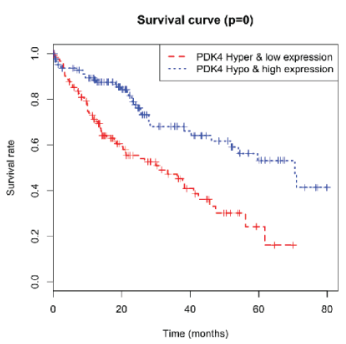

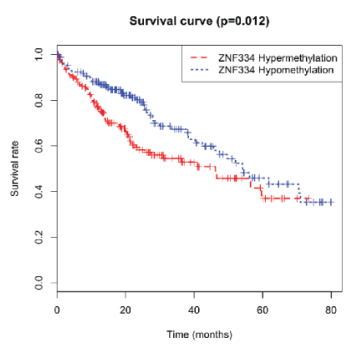

H

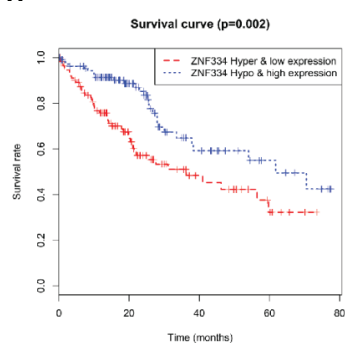

FIGURE 6 | Kaplan-Meier curves for methylation expression of MeDEGs are associated with overall survival. (A) CTF1, (B) FZD8, (C) PDK4 and (D) ZNF334 were ranked by the median of methylation and then scored for each patient in accordance with high- or low-level methylation value. (E) CTF1, (F) FZD8, (G) PDK4 and (H) ZNF334 were ranked by the median of methylation and expression and then scored for each patient in accordance with high- or low-level methylation value and high or low-level expression value. The horizontal axis represents the overall survival time and the vertical axis represents survival function.

(CCNE2), Frizzled-8 (FZD8), transforming growth factor beta-3 (TGFB3), laminin subunit alpha-2 (LAMA2), glutathione Stransferase $\mathrm{Mu} 1$ (GSTM1), and matrix metalloproteinase-2 (MMP2) are involved. Though RAC2 (20), GNB4 (21), PTGER4 (22, 23), CCNE2 (24), FZD8 (25), TGFB3 (26), LAMA2 (27), and GSTM1 $(28,29)$ have been reported to be regulated by methylation in multiple cancers, very little is known about the regulatory mechanisms by which methylation is involved in HCC. Moreover, the term "Hepatocellular carcinoma" is enriched with FZD8, ribosomal protein S6 kinase alpha-6 (PRSAKA6), TGFB3 and GSTM1. FZD8 has been demonstrated to be an important cell membrane receptor that mediates the Wnt signaling pathway in $\operatorname{HCC}(30,31)$. Feng et al. (32) have indicated that TGFB3 can function as a modulator to promote the metastatic phenotype of nonmetastatic HCC cells induced by TGFB1. GSTM1 polymorphisms have been identified as biomarkers of HCC development and risk in different regions (33-37).

In addition, methylation status of cardiotrophin-1 (CTF1), FZD8, pyruvate dehydrogenase kinase 4 (PDK4), and ZNF334 was associated with overall survival. Similar results were obtained by performing conjoint analysis of methylation,

TABLE 1 | Association between methylation of prognosis-related MeDEGs and clinical features.

\begin{tabular}{|c|c|c|c|c|c|c|c|c|c|c|c|c|}
\hline \multirow{2}{*}{$\begin{array}{l}\text { Variable } \\
\text { Age at diagnosis (yr) }\end{array}$} & \multicolumn{2}{|c|}{ CTF1 methylation } & \multirow[t]{2}{*}{$P$-value } & \multicolumn{2}{|c|}{ FZD8 methylation } & \multirow[t]{2}{*}{$P$-value } & \multicolumn{2}{|c|}{ PDK4 methylation } & \multirow[t]{2}{*}{$P$-value } & \multicolumn{2}{|c|}{ ZNF334 methylation } & \multirow[t]{2}{*}{$P$-value } \\
\hline & Low & High & & Low & High & & Low & High & & Low & High & \\
\hline$<60$ & 89 & 80 & 0.366 & 100 & 69 & $0.001^{\star \star}$ & 79 & 90 & 0.310 & 82 & 87 & 0.708 \\
\hline$\geq 60$ & 87 & 97 & & 76 & 108 & & 97 & 87 & & 94 & 90 & \\
\hline \multicolumn{13}{|l|}{ Gender } \\
\hline female & 46 & 65 & $0.043^{*}$ & 49 & 62 & 0.180 & 42 & 69 & $0.003^{\star \star}$ & 55 & 56 & 0.971 \\
\hline male & 130 & 112 & & 127 & 115 & & 134 & 108 & & 121 & 121 & \\
\hline \multicolumn{13}{|l|}{ T stage } \\
\hline $\mathrm{T} 1$ & 97 & 78 & $0.038^{*}$ & 92 & 83 & 0.368 & 100 & 75 & $0.009^{\star \star}$ & 91 & 84 & 0.489 \\
\hline T2-4 & 79 & 99 & & 84 & 94 & & 76 & 102 & & 85 & 93 & \\
\hline \multicolumn{13}{|l|}{$\mathrm{N}$ stage } \\
\hline NO & 173 & 170 & 0.202 & 171 & 172 & 0.993 & 174 & 169 & 0.055 & 170 & 173 & 0.741 \\
\hline N1 & 3 & 7 & & 5 & 5 & & 2 & 8 & & 6 & 4 & \\
\hline \multicolumn{13}{|l|}{ Pathologic stage } \\
\hline $1+\|$ & 138 & 124 & 0.094 & 134 & 128 & 0.485 & 140 & 122 & $0.031^{*}$ & 133 & 129 & 0.564 \\
\hline III + IV & 38 & 53 & & 42 & 49 & & 36 & 55 & & 43 & 48 & \\
\hline
\end{tabular}

${ }^{*} P<0.05,{ }^{* *} P<0.01$ 
TABLE 2 | Cox regression analyses of association between prognosis-related MeDEGs and clinicopathological characteristics.

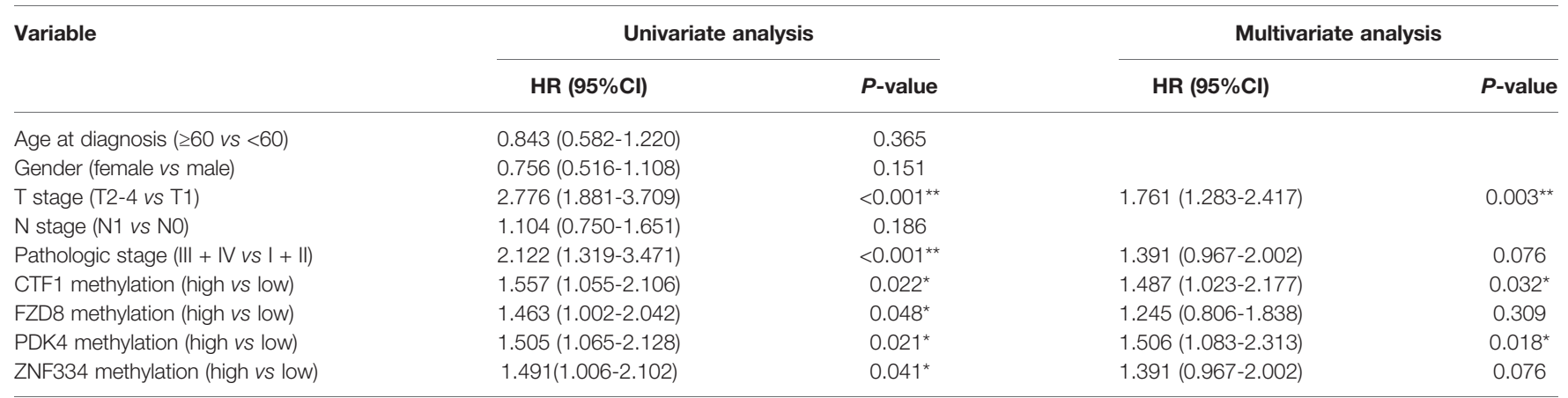

${ }^{*} P<0.05,{ }^{*} P<0.01$.

A

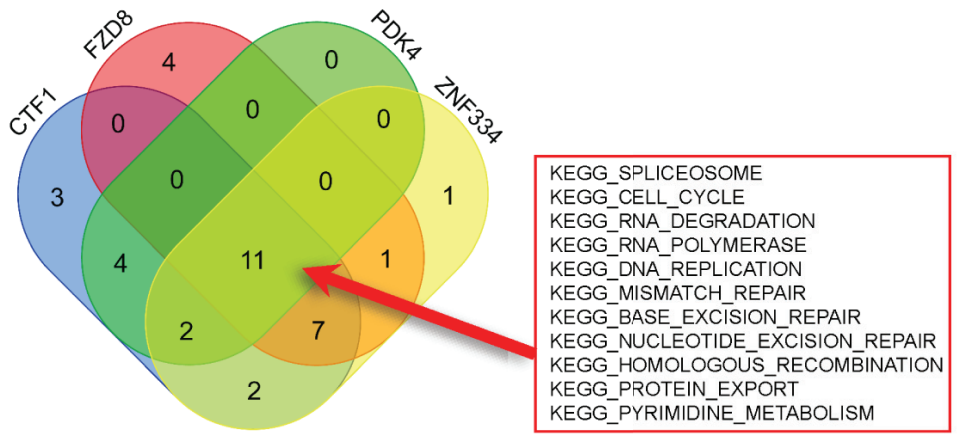

B

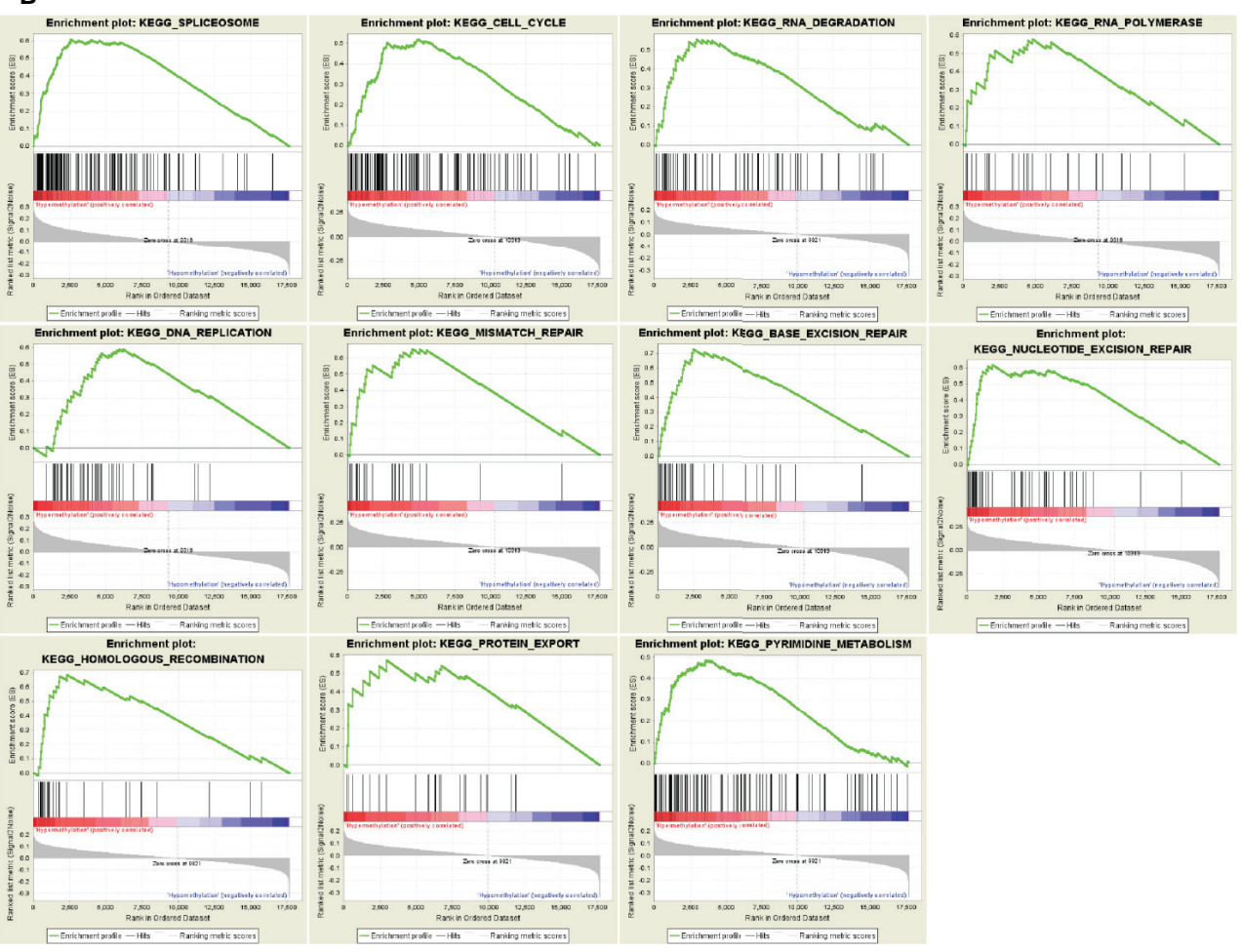

FIGURE 7 | Gene set enrichment analysis (GSEA) of prognosis-related MeDEGs. (A) Intersection of pathways enriched by hypermethylation of CTF1, FZD8, PDK4, and ZNF334 is shown in the diagram. (B) GSEA of PDK4 is shown as example. 
expression, and prognosis. CTF1 is a mitogenic cytokine of the interleukin 6 family, which is a hepatocyte survival factor that is up-regulated during liver regeneration in animal models (38). Bustos et al. (39) have indicated that CTF1 prevented colon cancer cell proliferation in the liver depending on T and NK cells. However, the function and regulatory mechanism of CTF1 in HCC remains controversial. A recent study has indicated that prognosis-related PDK4 is down-regulated in HCC tissues, while PDK4 knockdown promotes HCC cell proliferation, migration, and invasion (40). Moreover, arsenic-induced silencing of PDK4 in hepatic cells is mediated by histone $\mathrm{H} 3$ lysine 9 methylation in the promoter (41). ZNF334 lymphocyte expression can be regulated by tumor necrosis factor $\alpha$. However, little is known about ZNF334 in tumors (42). The present study first indicated that methylation of PDK4 and CTF1 is a potential independent biomarker for prognosis prediction. More studies are needed to verify this hypothesis.

GSEA clarified the mechanisms by which prognosis-related MeDEGs drive tumorigenesis. A total of 11 pathways that involved prognosis-related MeDEGs and the "spliceosome" pathway were the most significantly enriched. The spliceosome consists of five ribonucleoprotein subunits and protein cofactors and has been demonstrated as a critical and complicated mechanism in mRNA synthesis regulation of eukaryotic cells (43). Krogh et al. (44) have indicated that ribose methylation interrupts snRNA interactions and affects the splicing process in a $\mathrm{T}$ cell leukemia model. According to the GSEA results, almost every step of gene transcription and translation is enriched by methylation of prognosis-related MeDEGs.

The present study has some limitations. Firstly, the present research is mainly based on bioinformatic analysis of TCGA database and verification of identified genes and pathways is insufficient. Secondly, it is generally known that microsatelliteinstability (MSI) is associated with aberrant methylation in HCC.

\section{REFERENCES}

1. Bray F, Ferlay J, Soerjomataram I, Siegel RL, Torre LA. A. Jemal: Global Cancer Statistics 2018: GLOBOCAN Estimates of Incidence and Mortality Worldwide for 36 Cancers in 185 Countries. CA Cancer J Clin (2018) 68 (6):394-424. doi: 10.3322/caac.21492

2. Kulik L, El-Serag. Epidemiology HB. And Management of Hepatocellular Carcinoma. Gastroenterology (2019) 156(2):477-91. doi: 10.1053/ j.gastro.2018.08.065

3. Younossi ZM, Marchesini G, Pinto-Cortez H, Petta S. Epidemiology of Nonalcoholic Fatty Liver Disease and Nonalcoholic Steatohepatitis: Implications for Liver Transplantation. Transplantation (2019) 103(1):22-7. doi: 10.1097/tp.0000000000002484

4. White DL, Thrift AP, Kanwal F, Davila J, El-Serag HB. Incidence of Hepatocellular Carcinoma in All 50 United States, From 2000 Through 2012. Gastroenterology (2017) 152(4):812-20.e5. doi: 10.1053/j.gastro.2016.11.020

5. Dang S, Zhou J, Chen Y, Chen P, Ji M, Shi B, et al. Dynamic Expression of ZNF382 and its Tumor-Suppressor Role in Hepatitis B Virus-Related Hepatocellular Carcinogenesis. Oncogene (2019) 38(24):4804-19. doi: 10.1038/ s41388-019-0759-9

6. Sun N, Zhang J, Zhang C, Shi Y, Zhao B, Jiao A, et al. Using Illumina Infinium HumanMethylation 450k BeadChip to Explore Genomewide DNA Methylation Profiles in a Human Hepatocellular Carcinoma Cell Line. Mol Med Rep (2018) 18(5):4446-56. doi: 10.3892/mmr.2018.9441
Illumina Human Methylation 450K BeadChip data analyzed in the present research did not supply any MSI information. Thus, it is difficult to reveal the relationship between MSI, methylation and prognosis and more validation experiments are needed in the future.

In conclusion, MeDEGs were identified by analyzing the expression profiles and methylation data of HCC samples from TCGA database. GO and KEGG pathways analyses verified the MeDEG mechanisms. Furthermore, four prognosis-related MeDEGs and methylation status of PDK4 and CTF1 were identified as potential biomarkers for survival prediction and treatment.

\section{DATA AVAILABILITY STATEMENT}

The original contributions presented in the study are included in the article/supplementary material. Further inquiries can be directed to the corresponding author.

\section{AUTHOR CONTRIBUTIONS}

H-MY conducted the study. YL, BM, and PJ applied the experiments on TCGA project. YL wrote the manuscript. All authors contributed to the article and approved the submitted version.

\section{FUNDING}

The study was funded by the Revitalizing Liaoning Talents Program (grant number XLYC1907004).

7. Zhang Y, Petropoulos S, Liu J, Cheishvili D, Zhou R, Dymov S, et al. The Signature of Liver Cancer in Immune Cells DNA Methylation. Clin Epigenet (2018) 10:8. doi: 10.1186/s13148-017-0436-1

8. Wang Z, Jensen MA, Zenklusen JC. A Practical Guide to The Cancer Genome Atlas (Tcga). Methods Mol Biol (2016) 1418:111-41. doi: 10.1007/978-1-4939-3578-9_6

9. Midori H, Jennifer C, Amelia I, Jane L, Michael A, Russell C, et al. The Gene Ontology: The Gene Ontology (GO) Project in 2006. Nucleic Acids Res (2006) 34(Database issue):D322-6. doi: 10.1093/nar/gkj021

10. Draghici S, Khatri P, Tarca AL, Amin K, Done A, Voichita C, et al. A Systems Biology Approach for Pathway Level Analysis. Genome Res (2007) 17 (10):1537-45. doi: 10.1101/gr.6202607

11. Bindea G, Mlecnik B, Hackl H, Charoentong P, Tosolini M, Kirilovsky A, et al. ClueGO: A Cytoscape Plug-in to Decipher Functionally Grouped Gene Ontology and Pathway Annotation Networks. Bioinformatics (2009) 25 (8):1091-3. doi: 10.1093/bioinformatics/btp101

12. Mlecnik B, Galon J, Bindea G. Automated Exploration of Gene Ontology Term and Pathway Networks With Cluego-REST. Bioinformatics (2019) 35 (19):3864-66. doi: 10.1093/bioinformatics/btz163

13. Hu J, Duan B, Jiang W, Fu S, Gao H, Lu L. Epidermal Growth FactorContaining Fibulin-Like Extracellular Matrix Protein 1 (EFEMP1) Suppressed the Growth of Hepatocellular Carcinoma Cells by Promoting Semaphorin $3 \mathrm{~b}$ (Sema3b). Cancer Med (2019) 8(6):3152-66. doi: 10.1002/cam4.2144

14. Nanok C, Jearanaikoon P, Proungvitaya S, Limpaiboon T. Aberrant Methylation of HTATIP2 and UCHL1 as a Predictive Biomarker for 
Cholangiocarcinoma. Mol Med Rep (2018) 17(3):4145-53. doi: 10.3892/ mmr.2017.8319

15. Hinrichsen I, Kemp M, Peveling-Oberhag J, Passmann S, Plotz G, Zeuzem S, et al. Promoter Methylation of MLH1, Pms2, MSH2 and p16 is a Phenomenon of Advanced-Stage Hccs. PloS One (2014) 9(1):e84453. doi: 10.1371/journal.pone.0084453

16. Fan G, Tu Y, Chen C, Sun H, Wan C, Cai X. DNA Methylation Biomarkers for Hepatocellular Carcinoma. Cancer Cell Int (2018) 18:140. doi: 10.1186/s12935-018-0629-5

17. Zhang J, Liu Z, Lian Z, Liao R, Chen Y, Qin Y, et al. Monoacylglycerol Lipase: A Novel Potential Therapeutic Target and Prognostic Indicator for Hepatocellular Carcinoma. Sci Rep (2016) 6:35784. doi: 10.1038/srep35784

18. Zhao Q, Cai W, Zhang X, Tian S, Zhang J, Li H, et al. Rybp Expression is Regulated by KLF4 and Sp1 and Is Related to Hepatocellular Carcinoma Prognosis. J Biol Chem (2017) 292(6):2143-58. doi: 10.1074/jbc.M116.770727

19. Zhou J, Lai PB, Tsui SK. Identification of a Non-Coding KLF4 Transcript Generated From Intron Retention and Downregulated in Human Hepatocellular Carcinoma. Int J Oncol (2015) 47(4):1554-62. doi: 10.3892/ijo.2015.3104

20. Chen G, Wang Y, Wang L, Xu W. Identifying Prognostic Biomarkers Based on Aberrant DNA Methylation in Kidney Renal Clear Cell Carcinoma. Oncotarget (2017) 8(3):5268-80. doi: 10.18632/oncotarget.14134

21. Wang B, Li D, Rodriguez-Juarez R, Farfus A, Storozynsky Q, Malach M, et al. A Suppressive Role of Guanine Nucleotide-Binding Protein Subunit Beta-4 Inhibited by DNA Methylation in the Growth of Anti-Estrogen Resistant Breast Cancer Cells. BMC Cancer (2018) 18(1):817. doi: 10.1186/s12885-018-4711-0

22. Hiken JF, McDonald JI, Decker KF, Sanchez C, Hoog J, VanderKraats ND, et al. Epigenetic Activation of the Prostaglandin Receptor EP4 Promotes Resistance to Endocrine Therapy for Breast Cancer. Oncogene (2017) 36 (16):2319-27. doi: 10.1038/onc.2016.397

23. Weiss G, Schlegel A, Kottwitz D, Konig T, Tetzner R. Validation of the SHOX2/PTGER4 Dna Methylation Marker Panel for Plasma-Based Discrimination Between Patients With Malignant and Nonmalignant Lung Disease. J Thorac Oncol (2017) 12(1):77-84. doi: 10.1016/j.jtho.2016.08.123

24. Weng YP, Hung PF, Ku WY, Chang CY, Wu BH, Wu MH, et al. The Inhibitory Activity of Gallic Acid Against DNA Methylation: Application of Gallic Acid on Epigenetic Therapy of Human Cancers. Oncotarget (2018) 9 (1):361-74. doi: 10.18632/oncotarget.23015

25. Sonnet M, Claus R, Becker N, Zucknick M, Petersen J, Lipka DB, et al. Early Aberrant DNA Methylation Events in a Mouse Model of Acute Myeloid Leukemia. Genome Med (2014) 6(4):34. doi: 10.1186/gm551

26. Liu X, Qi J, Tao Y, Zhang H, Yin J, Ji M, et al. Correlation of Proliferation, TGF-beta3 Promoter Methylation, and Smad Signaling in MEPM Cells During the Development of ATRA-induced Cleft Palate. Reprod Toxicol (2016) 61:1-9. doi: 10.1016/j.reprotox.2016.02.010

27. Mello AA, Leal MF, Rey JA, Pinto GR, Lamarao LM, Montenegro RC, et al. Deregulated Expression of SRC, LYN and CKB Kinases by DNA Methylation and Its Potential Role in Gastric Cancer Invasiveness and Metastasis. PloS One (2015) 10(10):e0140492. doi: 10.1371/journal.pone.0140492

28. Wang SC, Huang CC, Shen CH, Lin LC, Zhao PW, Chen SY, et al. Gene Expression and DNA Methylation Status of Glutathione s-Transferase Mul and Mu5 in Urothelial Carcinoma. PloS One (2016) 11(7):e0159102. doi: 10.1371/journal.pone.0159102

29. Yeh SH, Liu CL, Chang RC, Wu CC, Lin CH, Yang. Aging-dependent DNA hypermethylation KD. And Gene Expression of GSTM1 Involved in T Cell Differentiation. Oncotarget (2017) 8(30):48591-602. doi: 10.18632/oncotarget.18109

30. Le PN, McDermott JD, Jimeno A. Targeting the Wnt Pathway in Human Cancers: Therapeutic Targeting With a Focus on OMP-54F28. Pharmacol Ther (2015) 146:1-11. doi: 10.1016/j.pharmthera.2014.08.005

31. Mebarki S, Desert R, Sulpice L, Sicard M, Desille M, Canal F, et al. De Novo HAPLN1 Expression Hallmarks Wnt-induced Stem Cell and Fibrogenic
Networks Leading to Aggressive Human Hepatocellular Carcinomas. Oncotarget (2016) 7(26):39026-43. doi: 10.18632/oncotarget.9346

32. Feng XX, Liu M, Yan W, Zhou ZZ, Xia YJ, Tu W, et al. Beta3 Integrin Promotes TGF-beta1/H2O2/HOCl-Mediated Induction of Metastatic Phenotype of Hepatocellular Carcinoma Cells by Enhancing TGF-beta1 Signaling. PloS One (2013) 8(11):e79857. doi: 10.1371/journal.pone.0079857

33. London WT, Evans AA, Buetow K, Litwin S, McGlynn K, Zhou T, et al. Molecular and Genetic Epidemiology of Hepatocellular Carcinoma: Studies in China and Senegal. Princess Takamatsu Symp (1995) 25:51-60.

34. Omer RE, Verhoef L, Van't Veer P, Idris MO, Kadaru AM, Kampman E, et al. Peanut Butter Intake, GSTM1 Genotype and Hepatocellular Carcinoma: A Case-Control Study in Sudan. Cancer Causes Control (2001) 12(1):23-32. doi: 10.1023/A:1008943200826

35. Chen J, Ma L, Peng NF, Wang SJ, Li LQ. A Meta-Analysis of the Relationship Between Glutathione S-transferases Gene Polymorphism and Hepatocellular Carcinoma in Asian Population. Mol Biol Rep (2012) 39(12):10383-93. doi: 10.1007/s11033-012-1917-0

36. Sophonnithiprasert T, Saelee P, Pongtheerat T. GSTM1 and GSTT1 Copy Number Variants and the Risk to Thai Females of Hepatocellular Carcinoma. J Gastrointest Oncol (2019) 10(2):324-29. doi: 10.21037/jgo.2018.09.14

37. Li S, Xue F, Zheng Y, Yang P, Lin S, Deng Y, et al. GSTM1 and GSTT1 Null Genotype Increase the Risk of Hepatocellular Carcinoma: Evidence Based on 46 Studies. Cancer Cell Int (2019) 19:76. doi: 10.1186/s12935-019-0792-3

38. Bustos M, Beraza N, Lasarte JJ, Baixeras E, Alzuguren P, Bordet T. Protection Against Liver Damage by cardiotrophin-1: A Hepatocyte Survival Factor UpRegulated in the Regenerating Liver in Rats. Gastroenterology (2003) 125 (1):192-201. doi: 10.1016/S0016-5085(03)00698-X

39. Bustos M, Dubrot J, Martinez-Anso E, Larequi E, Castano D, Palazon A, et al. Cardiotrophin-1 Determines Liver Engraftment of Syngenic Colon Carcinoma Cells Through an Immune System-Mediated Mechanism. Oncoimmunology (2012) 1(9):1527-36. doi: 10.4161/onci.22504

40. Yang C, Wang S, Ruan H, Li B, Cheng Z, He J, et al. Qin and H. Jin: Downregulation of PDK4 Increases Lipogenesis and Associates With Poor Prognosis in Hepatocellular Carcinoma. J Cancer (2019) 10(4):918-26. doi: $10.7150 /$ jca. 27226

41. Zhang X, Wu J, Choiniere J, Yang Z, Huang Y, Bennett J, et al. Arsenic Silences Hepatic PDK4 Expression Through Activation of Histone H3K9 Methylatransferase G9a. Toxicol Appl Pharmacol (2016) 304:42-7. doi: 10.1016/j.taap.2016.05.015

42. Henc I, Soroczynska-Cybula M, Bryl E, Witkowski JM. The In Vitro Modulatory Effect of TNFalpha on the mRNA Expression and Protein Levels of Zinc Finger Protein ZNF334 in CD4(+) Lymphocytes of Healthy People. Acta Biochim Pol (2015) 62(1):113-7. doi: 10.18388/abp.2014_857

43. Matera AG, Wang Z. A Day in the Life of the Spliceosome. Nat Rev Mol Cell Biol (2014) 15(2):108-21. doi: 10.1038/nrm3742

44. Krogh N, Kongsbak-Wismann M, Geisler C, Nielsen H. Substoichiometric Ribose Methylations in Spliceosomal Snrnas. Org Biomol Chem (2017) 15 (42):8872-76. doi: 10.1039/c7ob02317k

Conflict of Interest: The authors declare that the research was conducted in the absence of any commercial or financial relationships that could be construed as a potential conflict of interest.

Copyright (C) 2021 Liang, Ma, Jiang and Yang. This is an open-access article distributed under the terms of the Creative Commons Attribution License (CC BY). The use, distribution or reproduction in other forums is permitted, provided the original author(s) and the copyright owner(s) are credited and that the original publication in this journal is cited, in accordance with accepted academic practice. No use, distribution or reproduction is permitted which does not comply with these terms. 